\title{
Advanced Practice Nurse-managed Heart Failure Clinic Benefits Patient's Quality of Life and Limits Readmissions
}

\author{
Christina Hanson, MSN, ACNS-BC ${ }^{1, *}$, Kara Aalfs, MSN, ACNS-BC ${ }^{2}$, \\ Donna J. Plonczynski, PhD, APRN, FNP-BC ${ }^{3}$ \\ ${ }^{1}$ Clinical Nurse Specialist Heart Failure Recovery Center, Advocate Sherman Hospital Elgin, 60124 Illinois, United States \\ ${ }^{2}$ Manager of Out-Patient Cardiovascular Services, Advocate Sherman Hospital Elgin, 60124 Illinois, United States \\ ${ }^{3}$ Northern Illinois University DeKalb, Illinois, United States \\ *Corresponding Author: First Author Christina.Hanson@advocatehealth.com
}

Copyright (C) 2013 Horizon Research Publishing All rights reserved.

\begin{abstract}
Background: Heart failure (HF) is the primary cause of hospitalizations and readmissions to a hospital within 30 days of initial discharge. In $2010 \mathrm{HF}$ cost an estimated $\$ 39.2$ billion in health care services, lost productivity and medications. Optimizing outpatient care is vital to effectively manage this patient population. Objective: The purpose of this paper is to describe and evaluate the effectiveness of an optimized outpatient HF clinic. Patient outcomes from a Midwestern community hospital were based on quality of life (QOL) and readmission rates. Methods: A retrospective chart audit was conducted on a random sample of patients enrolled in the Heart Failure Recovery Center (HFRC). The QOL outcomes were measured using the Minnesota Living with Heart Failure ${ }^{\circledR}$ Questionnaire. Results: Enrolled patients demonstrated improvement in their QOL in the emotional, physical, and overall scores across the first 6 months of care. In addition, there were significantly fewer 30 -day all-cause readmissions when compared to non-Clinic patients. Conclusion: Utilization of an APN-managed multidisciplinary HF clinic improved patient's QOL and contributed to one hospital's decreased all-cause readmission rates for the HF population.
\end{abstract}

Keywords Heart Failure, Advanced Practice Nurse, Readmissions, Quality of Life

\section{Introduction}

Every year there are over 670,000 people diagnosed with heart failure (HF) in the United States, which is the most rapid growing cardiac diagnosis 1 . Currently, there are an estimated 6.6 million Americans living with HF 1. These incidence and prevalence rates are projected to continue to climb as the country's aging population reaches record numbers 2 .

The American Heart Association (AHA) predicts that by $2030,40 \%$ of the US population will have a cardiovascular disease (CVD) diagnosis 3. It is estimated that the direct cost of cardiovascular diseases will be $\$ 95$ billion annually, which does not account for indirect costs such as lost productivity 1 . Specifically for HF, the AHA estimates costs in 2015 to be as high as 44.6 billion dollars annually, which will more than double in the next 15 years.

For adults over the age of 65 years, HF is the most frequent diagnosis for hospitalization and is linked to high re-hospitalization rates, which contributes to high costs for patients, families, and society 1 . The Centers for Medicare and Medicaid Services (CMS) has placed great emphasis on the reduction in hospital readmission rates of HF patients. These rates are important quality indicators that are measured by outcomes, indicating patient safety and effective care 4.

As the incidence rate of HF continues to rise, hospitals are looking for ways to improve patient outcomes and reduce cost. More research is needed to determine how to most effectively manage the continuum of care from outpatient and inpatient care models for adults with HF 5. Because successful management requires significant lifestyle changes, multidisciplinary healthcare team members can contribute to the improvement of each patient's quality of life (QOL) 6, 7.

\section{Objectives}

The purpose of this paper is twofold. First, the paper will describe the process of an optimized outpatient APN-managed clinic for HF patients from a Midwestern community hospital. Secondly, the exploration of the outcomes of the clinic on QOL and readmission rates will be outlined.

\section{Methods}

The Heart Failure Recovery Center (HFRC) is an outpatient Clinic located in the Northwest suburbs of 
Chicago within a 250-bed community-hospital. The primary objectives of the HFRC are to improve clinical outcomes by improving each patient's QOL and reducing hospital readmissions by the use of evidence based practice (EBP). At each HFRC visit, the APN performs a cardiac focused assessment and formulates a plan of care for optimizing patient outcomes. The Clinic offers comprehensive services to patients including collaboration between the patient's healthcare team members and provides an individualized education and motivational plan.

An integrated multidisciplinary team of APN's, pharmacists, physicians, rehabilitation therapists, case managers, home health nurses, and a nutritionist work together to educate and care for the HF patient population. The patient is referred by an attending physician for enrollment into the Clinic. For patients referred to the Clinic while hospitalized, the APN coordinates a follow-up appointment within 7-10 days after discharge. Each component of the HFRC is described in Figure 1. After the patient has completed 4 visits, follow-up appointments to the Clinic are scheduled at least on a monthly basis. The APN may follow patients more closely based upon the individual patient needs and clinical judgment.

The initial visit begins with an introduction to the HFRC, the emphasis of which is 'recovery'. The APN establishes the patient's medical history, medical management plan, and reviews all diagnostic tests. The assessment includes a focused cardiovascular physical exam, followed by an evaluation of cardiac function. The assessment further includes an evaluation of the patient's resources, strengths and weaknesses, coping behaviors, and the impact of the environment upon the patient. One week later, the next visit includes a consultation with a pharmacist to address medications. Objectives of this consultation include identifying the purpose of each medication, common side effects and proper medication regimen for HF patient population. Furthermore, patients verbalize the dosing schedule of each medication and the pharmacist assesses the patient's cost-effective access to medications during this session. The meeting with the pharmacist is immediately followed by further assessment by the APN. This session includes depression screening and sleep apnea screening. The depression screening tool used is the Harvard Department of Psychiatry/National Depression Screening Day Scale (HANDS) 11. The depression screening tool identifies symptoms of depression based on a 30-point scale through a series of ten questions. Additionally, the screening tool used to identify sleep apnea includes nine questions assessing snoring, collar size, and tiredness. Based on the results of these screening tools, patients are referred to the appropriate department for follow up.

Figure 1. Components of Heart Failure Recovery Center

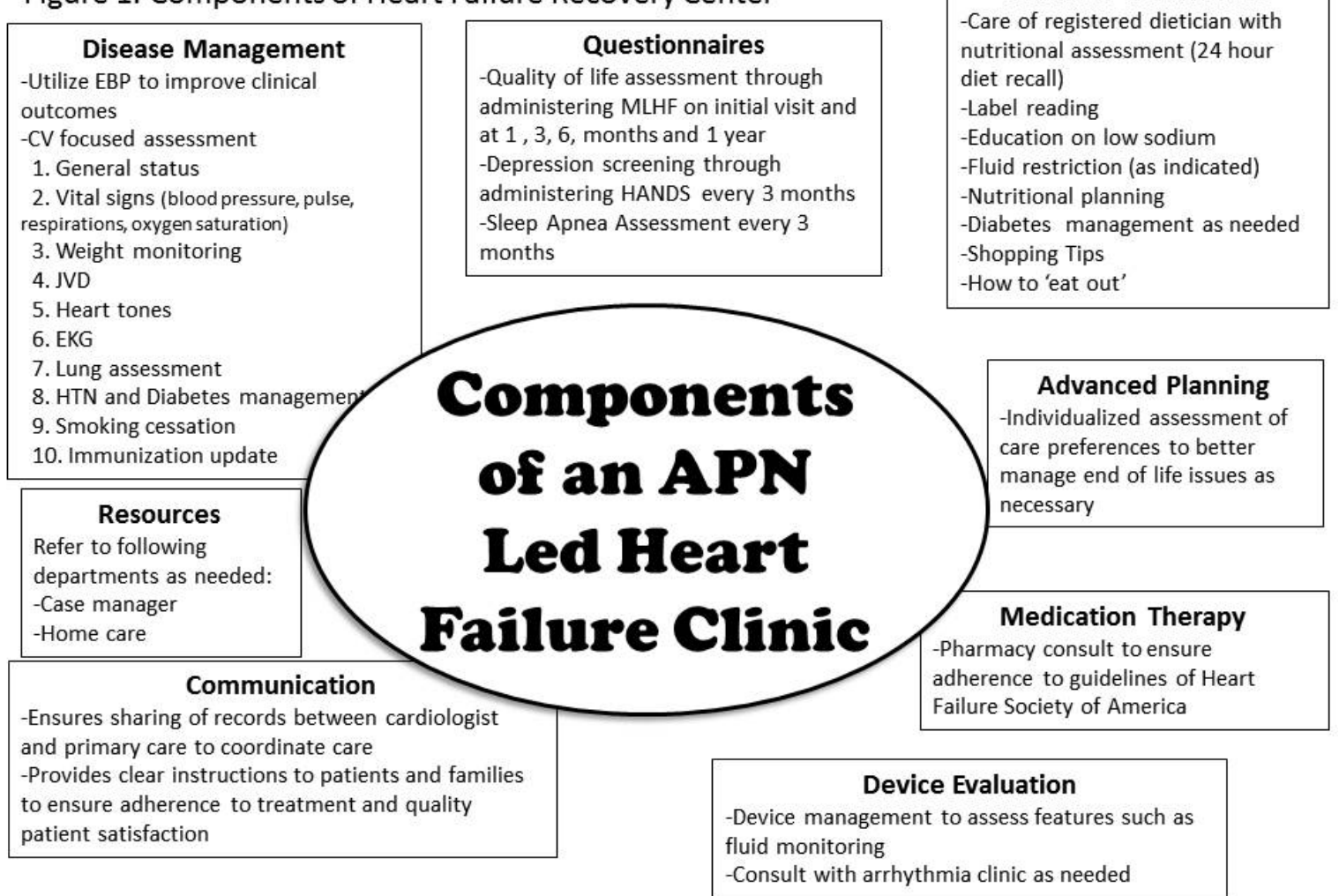

Note: $\mathrm{EBP}=$ Evidence Based Practice, $\mathrm{CV}=$ Cardiovascular, JVD= Jugular venous distention, MLHF= Minnesota Living with Heart Failure Questionnaire, HANDS= Harvard Department of Psychiatry/National Depression Screening Day Scale

Figure 1. Components of Heart Failure Recovery Center 
The third appointment involves a consultation with a registered nutritionist to conduct a nutritional history and plan. In addition, there is a tailored discussion regarding healthy eating when living with HF in accordance with the American Dietetic Association's guidelines 12

After the third appointment, the APN continues to follow the patient's management with a comprehensive care plan. The frequency of future HFRC visits are made per the clinical judgment of the APN, but will be scheduled at least every 4-to-6 weeks. Case management and home care resources are arranged as needed throughout the patient's enrollment in the HFRC. Other resources available to the Clinic include cardio-pulmonary rehabilitation to aid HF patients enhance everyday activity. In addition to these services, more intensive education is available from any of the team members.

Because communication among healthcare providers is essential to provide comprehensive care to these complex patients, the APN's clinical notes for each visit are filed within the hospital's computerized charting system for all interdisciplinary team members to access. In addition, a copy of the Clinic progress note is sent directly to the referring physician and/or cardiologist and primary care physician.

Secondly, the primary analysis was to compare trends in QOL over time in a population that has usual decline in this measure. The secondary analysis was a comparison of community dwelling adults with usual care to the population receiving multidisciplinary care in the Clinic, which was coordinated by the APN. Usual care in the community does not include individualized counseling or case management, thought education may be conducted at various levels. Because this study was retrospective, the authors were not able to control the amount of care provided in the usual medical practices.

\section{Instruments}

During the initial clinic visit, the APN administers The

Table 2. Mean, Standard Deviation, and Range of Quality of Life scores by Dimension and Time from chart audit

\begin{tabular}{|c|c|c|c|c|c|}
\hline \multirow{2}{*}{ Dimension } & Time & Mean & Std. Deviation & Minimum score & Maximum score \\
\hline \multirow{3}{*}{ Total } & Baseline & 49.85 & 23.180 & 5 & 84 \\
\cline { 2 - 6 } & 1 month & 33.40 & 19.307 & 1 & 66 \\
\cline { 2 - 6 } & 6 months & 22.20 & 17.867 & 0 & 33 \\
\hline \multirow{2}{*}{ Physical } & Baseline & 22.80 & 11.270 & 0 & 33 \\
\cline { 2 - 6 } & 1 month & 16.55 & 8.775 & 0 & 31 \\
\cline { 2 - 6 } & 6 months & 10.05 & 7.647 & 0 & 25 \\
\hline \multirow{2}{*}{ Emotional } & Baseline & 10.80 & 6.925 & 0 & 24 \\
\cline { 2 - 6 } & 1 month & 7.50 & 6.436 & 6.656 & 0 \\
\hline
\end{tabular}

Minnesota Living with Heart Failure ${ }^{\circledR}$ Questionnaire (MLHF) to assess QOL 13-15. The questionnaire includes 21 questions, eight of which are in relation to the physical dimension of $\mathrm{HF}$ and five questions are in relation to the emotional dimension. This valid tool is utilized to assess the patient's baseline quality of life and then it is reassessed at 1 month, 3 months, 6 months, and 1 year.

In order to evaluate the effectiveness of the Clinic, two retrospective audits were conducted, which included the charts from the population of 153 patients examined in the outpatient HFRC for one year. After IRB approval, charts were randomly selected and included in the analysis if they contained at least 3 longitudinal measures of the MLHF questionnaire within the first 6 months of treatment in the Clinic. Data from baseline, 1 month and 6 months were used in the analysis.

Table 1. Demographics of Heart Failure Clinic compared to hospital patients

\begin{tabular}{|c|c|c|c|c|}
\hline & $\begin{array}{c}\text { Mean } \\
\text { age }\end{array}$ & Patients & Gender & $\begin{array}{c}\text { Readmission } \\
\text { Rate }\end{array}$ \\
\hline $\begin{array}{c}\text { Heart } \\
\text { Failure } \\
\text { Clinic }\end{array}$ & 71.25 & 20 & $\begin{array}{c}\text { Male 13 (65\%) } \\
\text { Female 7 (35\%) }\end{array}$ & $1 \%$ \\
\hline Hospital & 80.3 & 71 & $\begin{array}{c}\text { Male 35 (49\%) } \\
\text { Female 36(51\%) }\end{array}$ & $17.2 \%$ \\
\hline
\end{tabular}

\section{Results}

SPSS version 18 was used in the analysis and a p-value of $<.05$ was set as the significance level. Demographics of audited charts of patients in the Clinic are displayed in Table 1 in order to compare Clinic to hospitalized patients, with same diagnosis. 
Quality of life outcome measures with mean scores, standard deviations and range by dimension and time are displayed in Table 2. A repeated measures ANOVA was used to analyze the differences by dimension over time. Mean scores on all three dimensions decreased consistently during the first 6 months of care at the Clinic. Lowered scores are associated with improved QOL. Significant improvements were made in overall QOL score between times $(\mathrm{F}=9.44(2,57), \mathrm{p}<.001)$. This same improvement was measured in Physical $(\mathrm{F}=9.29(2,57), \mathrm{p}<.001)$ and Emotional dimensions $(\mathrm{F}=4.12(2,57), \mathrm{p}<.05)$.

For the evaluation of readmission rates, we compared complete annual data available. For one year there were 71 patients readmitted to the hospital within 30 days for the diagnosis of HF. The readmission rate for patients enrolled in the APN-managed HF Clinic was $1 \%$ compared to the community hospital's 30 day all-cause readmission rate for HF of $17.2 \%$.

\section{Discussion}

This paper identifies significant improvements in the HF patient's QOL overall and within the physical and emotional dimensions. This finding corresponds with a study that examined APN-coordinated care of aging patients transitioning from the hospital setting to home, which contributed to reduced readmission rates, a significant cost savings, improved QOL and improved physical functioning 16.

While it was anticipated that the readmission rates for the population served by the Clinic were better than those patients discharged to usual care, the difference in recidivism rates was remarkable. These findings have been identified in other literature, but not to this magnitude 17 . Of note is the 30-day readmission rate for Medicare patients with HF, which nationally is $24.8 \% 18$. Therefore, while the hospital has an improved rate over the national average, the Clinic results compare quite favorably.

\section{Future practice considerations}

The most rapidly growing cardiac diagnosis is HF. APNs are in an ideal role to monitor HF symptoms and educate patients to manage their chronic symptoms. As hospitals strive to reduce readmissions in order to ensure quality care and reduce healthcare costs, a comprehensive approach to managing HF can be managed by APNs. Continued research is needed to support the effectiveness of APN services to improve patient outcomes and expand their scope.

\section{Conclusion}

As the United States aging population continues to grow it is anticipated the prevalence of this chronic illness will continue to rise 1. The Heart Failure Society of America guidelines place a great emphasis on self-care and enhancing healthcare, which is at the core of the HFRC's success. APNs provide quality care while managing the multidisciplinary team to prevent and treat HF patients with an overall reduction in healthcare costs. The emphasis on 'recovery' and empowerment are the mainstays of the care provided.

This paper recognizes the limitation of the results to one community hospital over a one-year measure with limited patients. However, the results were highly significant and consistent with similar findings of APN-managed outpatient care outcomes.

\section{REFERENCES}

[1] Roger VL, Go AS, \& Lloyd-Jones D, et al. Heart disease and stroke statistics-2012 update. A report from the American Heart Association. Circulation. 2012;125:2-220.

[2] The Next Four Decades the Older Population in the United States: 2010 to 2050. Population Estimates and Projections US Census Bureau. Current Population Reports. Updated 2010. http://www.census.gov/prod/2010pubs/p25-1138.pdf Accessed May 24, 2012.

[3] Heidenreich PA, Trogdon JG, Khavjou OA, et al. Forecasting the future of cardiovascular disease in the United States: a policy statement from the American Heart Association. Circulation. 2011; 123(8):933-44.

[4] Medicare Physician Group Practice Demonstration. Centers for Medicare \& Medicaid Services Updated 2011. http://www.cms.gov/DemoProjectsEvalRpts/downloads/PGP _Fact_Sheet.pdf. Accessed on May 24, 2012.

[5] Manning S. Bridging the gap between hospital and home a new model of care for reducing readmission rate in chronic heart failure. Journal of Cardiovascular Nursing. 2011; 26(5): 368-376.

[6] Pressler SJ, Subramanian U, Perkins SM, et al. Measuring depressive symptoms in heart failure: Validity and reliability of the health questionnaire. American Association of Critical-Care Nurses. 2011; 20:146-152.

[7] Allen J \& Dennison C. Randomized trials of nursing interventions for secondary prevention in patients with coronary artery disease and heart failure systematic review. Journal of Cardiovascular Nursing.2010; 25(3):207-220.

[8] Hauptman PJ, Rich MW, Heidenreich PA, et al. The heart failure clinic: A consensus statement of the Heart Failure Society of America. JCardfail. 2008;14(10):801-815.

[9] Jessup M, Abraham WT, \& Casey, DE, et al. 2009 Focused update: ACCF/AHA guidelines for the diagnosis and management of heart failure in adults. Circulation. 2009; 119:1977-2016.

[10] Beck C. Research on health-related quality of life and cardiac conditions. Home Health Nurse. 2012;30(1):54-60.

[11] Baer L, Jacobs DG, Meszler-Reizes J, et al. Development of a brief screening instrument: the HANDS. Psychother Psychosom. 2000;69:35-41.

[12] Heart Failure Evidence-Based Nutrition Practice Guidelines. American Dietetic Association Updated 2011. 
http://www.adaevidencelibrary.com/topic.cfm?cat=3249 Accessed on March 3, 2012.

[13] Statement on Clinical Nurse Specialist Practice and Education 2004 published by National Association of Clinical Nurse Specialists, Harrisburg, PA.

[14] Dahl J, \& Penque S. APN spells success for a heart failure program. Nursing Management. 2002;33(2), 46-48.

[15] Rector TS, Francis GS, Cohn JN. Patient's self-assessment of their congestive heart failure. Part 1 patient perceived dysfunction and its poor correlation with maximal exercise test. Heart Failure. 1987;Oct/Nov:192-196.
[16] McCauley KM, Bixby B, Naylor MD. Advanced practice nurse strategies to improve outcomes and reduce cost in elders with heart failure. Disease Management. 2006;9(5):302-310.

[17] Rich MW, Beckman V, Wittenberg C, Leven CL, Freeland $\mathrm{KE}$, Carney RM. A multidisciplinary intervention of prevent the readmission of elderly patients with congestive heart failure. N Engl J Med. 1995;333:1190-1195.

[18] US Department of Health and Human Services. Readmission Tables. Last updated March 3, 2012. Accessed on May 24, 2012http://www.hospitalcompare.hhs.gov/hospital-profile.as px?pid $=140030 \&$ lat $=41.87782 \& \operatorname{lng}=-88.33365 \& \#$ OOC 